\title{
Biotin Deficiency as a Target for Treating Restless Legs Syndrome in Chronic Dialysis Patients

\author{
Heidi Moretti ${ }^{1 *}$, Nell Matthews ${ }^{2}$, John David Anthony Lakatua', Tammy Keeling-Hathaway ${ }^{1}$ and Donald M Mock ${ }^{2,3}$
}

${ }^{1}$ Saint Patrick Hospital and Health Sciences Center, Missoula, MT, USA

${ }^{2}$ Department of Biochemistry, University of Arkansas for Medical Sciences, Little Rock AR, USA

${ }^{3}$ Department of Pediatrics, University of Arkansas for Medical Sciences, Little Rock AR, USA

\begin{abstract}
Restless Legs Syndrome is a neuropathic disorder seen in end stage renal disease. Since biotin is dialyzable, we examined the relationship between biotin status and Restless Legs Syndrome in end stage renal disease (Study 1) and determined the effect of biotin supplementation on Restless Legs Syndrome symptoms (Study 2). Comparison of prevalence of biotin deficiency in those with and without Restless Legs Syndrome (Study 1) and randomized assignment, double-blinded, placebo-controlled study of biotin supplementation (Study 2) were in a medical center's outpatient dialysis unit. In Study 1, patients received chronic dialysis and routine biotin supplementation at $\approx 300 \mu \mathrm{g}$ daily (10 times the recommended dietary allowance). In the intervention Study 2, biotin was supplemented at 10,000 $\mu \mathrm{g}$ daily for 8 weeks; the placebo group received $\approx 300 \mu \mathrm{g}$ of biotin daily. Outcome was measured by Restless Legs Syndrome symptom score and biotin status as indicated by activation coefficient of the biotin-dependent enzyme propionyl-CoA carboxylase in peripheral blood lymphocytes. In Study 1, patients with $(n=30)$ were more likely than those without $(n=19)$ Restless Legs Syndrome to be biotin deficient as judged by increased activation coefficient of propionyl-CoA carboxylase; mean $( \pm 1 \mathrm{SD})$ was $1.17 \pm 0.65$ vs. $0.87 \pm 0.39$, respectively $(P=0.007)$. In Study 2 , Restless Legs Syndrome score of the biotin group ( $n=16)$, improved from $20 \pm 6$ to $13 \pm 11(P=0.001)$. However, in the placebo group $(n=15)$, Restless Legs Syndrome score also improved from $17 \pm 8$ to $12 \pm 10(P=0.01)$. Consequently, this change was not different between biotin and placebo. Mean activation coefficient of propionyl-CoA carboxylase of the biotin group $(n=8)$ improved from $1.38 \pm 0.76$ to $0.81 \pm 0.25(P=0.01)$ and did not change in the placebo group $(0.98 \pm 0.44$ vs. $1.17 \pm 0.32 ; P=0.17)$. The improvement of biotin status and of Restless Legs Syndrome score with the $10,000 \mu \mathrm{g}$ supplement suggests that large biotin supplements might be necessary to optimize biotin status in dialysis patients.
\end{abstract}

Keywords: Dialysis; Restless Legs Syndrome; Biotin; Vitamin supplementation; End stage renal disease

Abbreviations: RLS: Restless Legs Syndrome; ESRD: End Stage Renal Disease; AC: Activation Coefficient; PCC: Propionyl-CoA Carboxylase; AI: Adequate Intake; IRLS: International Restless Legs Syndrome Study

\section{Introduction}

The prevalence of Restless Legs Syndrome (RLS) among dialysis patients in the United States has been reported to be as little as $20 \%$ [1] and as great as $68 \%$ [2]. In contrast, the prevalence in the general population is $0.4-2.5 \%[3,4]$. The primary pathogenesis remains unknown, but several mechanisms have been proposed including uremic peripheral neuropathy and side effects of dialysis including functional deficiencies of several micronutrients such as iron [5] potentially exacerbated by dialysis [6]. Of particular note, two reports of open label biotin supplementation at $10,000 \mu \mathrm{g}$ /day for 1 to 3 months in patients with End Stage Renal Disease (ESRD) receiving dialysis therapy concluded that the clinical features of peripheral neuropathy including RLS symptoms improved with biotin [7-9]. In one of the studies, profound improvement was reported in all participants; improved neurologic findings included orientation, speech, memory, myoclonic jerks, flapping tremor, restless legs, paresthesia, hiccups, and ambulation [8].

Biotin is a water-soluble vitamin that has multiple roles in the intermediary metabolism of carbohydrates, fats, and amino acids arising from the role of biotin as an essential, covalently-bound cofactor for five carboxylases [10]. Propionyl-CoA carboxylase (PCC) is a mitochondrial carboxylase that requires covalently bound biotin as a cofactor. Reduced PCC activity in blood lymphocytes reflects reduced intracellular biotin status in humans in whom biotin deficiency has been induced experimentally [11]. Another measure of tissue biotin status is the activation coefficient (AC) of PCC: the ratio of PCC activity after incubation of intact, viable blood lymphocytes with biotin to the activity of PCC in the same sample before incubation with biotin. The increase in the AC of PCC reflects biotin transport into the lymphocyte and incorporation in an existing mass of apo-PCC [12]. The increase of the AC during marginal biotin deficiency reflects the intracellular accumulation of apo-PCC.

Biotin is likely substantially depleted by both hemodialysis and peritoneal dialysis because of its small (244.31 Daltons) size and presence in the aqueous phase $[13,14]$. However, biotin is now routinely given to dialysis participants at $150-300 \mu \mathrm{g}$ per day orally, which is 5-10 times the recommended Adequate Intake (AI) for healthy adults [15]. In these complex circumstances, we hypothesized that suboptimal biotin status might contribute to the pathogenesis of RLS in some dialysis patients. In an initial study in individuals with ESRD receiving chronic dialysis (Study 1), we compared biotin status in those with RLS to those without. In a follow up study (Study 2), we evaluated

${ }^{*}$ Corresponding author: Heidi Moretti, MS, RD, Clinical Dietitian, Saint Patrick Hospital, 500 W. Broadway, Missoula, MT 59802, USA, Tel: 406-546-9481; Fax 406-327-1766; E-mail: hmoretti@saintpatrick.org

Received August 30, 2012; Accepted November 15, 2012; Published Novembe 23, 2012

Citation: Moretti H, Matthews N, Anthony Lakatua JD, Keeling-Hathaway T, Mock DM (2012) Biotin Deficiency as a Target for Treating Restless Legs Syndrome in Chronic Dialysis Patients. J Nephrol Therapeut 2: 128. doi:10.4172/21610959.1000128

Copyright: $\odot 2012$ Moretti H, et al. This is an open-access article distributed under the terms of the Creative Commons Attribution License, which permits unrestricted use, distribution, and reproduction in any medium, provided the original author and source are credited. 
whether a very large biotin supplement would improve RLS symptoms and whether indicators of biotin deficiency would improve with biotin supplementation despite pre-existing and continuing standard of care supplementation with biotin at several times the AI.

\section{Methods}

\section{Participants}

This study was approved by the institutional review boards of the Saint Patrick Hospital and Health Sciences Center and the University of Arkansas for Medical Sciences. Written, informed consent was obtained from all participants as part of an ongoing consent process. All participants were recruited from the Saint Patrick Hospital dialysis unit. All biotin analyses were conducted at the University of Arkansas for Medical Sciences.

Inclusion criteria included ESRD requiring hemodialysis or peritoneal dialysis therapy for duration of at least 3 months. Individuals with a history of consumption of more than $300 \mu \mathrm{g}$ daily of biotin were excluded. All participants continued their current medications under the direction of their physician including any medications for RLS. All were receiving a daily multivitamin containing either 150 or $300 \mu \mathrm{g}$ of biotin.

\section{Diagnosis of restless legs syndrome}

RLS was diagnosed prior to enrollment; the diagnosis was made by the participants' primary care physicians based on clinical symptoms, including: 1) urge or need to move the legs, usually accompanied or caused by unpleasant sensations; 2) symptoms are worse at rest; 3) Symptoms are at least partially and temporarily relieved by activity; and 4) symptoms show a circadian pattern, with a maximum in the evening or at night.

All participants with the diagnosis of RLS had an International Restless Legs Syndrome Study (IRLS) Group [16] rating scale score of 4 or more at the time of enrollment confirming the diagnosis of RLS. This scoring tool has been shown to be uniform with high reliability and validity [16]. The IRLS score was calculated using IRLS investigator version 2.2, which calculates IRLS score using a 10-item scale that assesses the severity of sleep disturbances as well as frequency and severity of restless legs symptoms. Copyright permission was obtained through Mapi Research Trust for use of the IRLS scoring tool.

\section{Sample collection and processing}

Heparinized blood was collected from in-dwelling catheter lines in ESRD participants. Blood lymphocytes were immediately isolated by density gradient as previously described [11] and divided into two equal samples. One was incubated with $10 \mathrm{nmol} / \mathrm{L}$ of biotin at room temperature for $30 \mathrm{~min}$ to activate apo-PCC as previously described [12]. The other was held at room temperature for the same time interval; then both aliquots were processed and stored frozen at $-80^{\circ} \mathrm{C}$ as previously described [12], shipped to UAMS, and PCC activity was measured as described below.

Activity of PCC in blood lymphocytes was determined by ${ }^{14} \mathrm{C}-\mathrm{HCO}_{3}$ incorporation assay [17]. The fundamental principle of the assay is quantitation of ${ }^{14} \mathrm{C}$ incorporation into acid-precipitable methylmalonyl CoA from ${ }^{14} \mathrm{C}$-bicarbonate, a substrate of PCC. The PCC must remain catalytically active after sample processing, cell isolation, and storage. Accordingly, the blood samples require prompt handling, the lymphocytes must be carefully isolated from blood, and the cell isolates must be stored frozen. These ideal conditions were present for the validation studies of PCC and AC of PCC, which were conducted in a Clinical Research Center $[11,12,18]$. However, the unavoidable variability of timing of sample availability for Study 1 and Study 2 has the potential to produced artifactual decreases in absolute PCC activity. An important advantage of the AC of PCC, which is a ratio of enzyme activity for samples handled contemporaneously in any given participant, is that enzyme instability artifacts are normalized by calculating a ratio of PCC activities for samples handled identically (other than incubation of the lymphocytes with a buffer that does or does not contain biotin).

As described previously, the PCC assay was performed with and without biotin in triplicate for each sample $[11,12,18]$. Activity was normalized by protein concentration of the suspension as determined by bicinchoninic acid protein assay as previously described $[11,12,18]$. Care must be taken during the processing and isolation steps of sample preparation because any hemoglobin contamination from red blood cells in the lymphocyte pellet interferes with the normalization of the PCC activity by lymphocyte protein. To avoid artifacts introduced by differences in efficiency of lymphocyte isolation ("stickiness" of lymphocytes which vary from participant to participant) and differences in hemoglobin contamination, protein content was determined from subaliquots of the lymphocyte suspensions incubated with biotin and without biotin. The activation coefficients were calculated as the ratio of PCC in lymphocytes with biotin activation to PCC activity in lymphocytes without biotin activation [12]. For AC of PCC, the normal range of $0.85-1.37$ was based on the full range observed in thirteen biotin-sufficient, healthy adults $[12,19]$.

\section{Biotin supplementation in Study 2}

We supplemented participants in the treatment arm of Study 2 with $5000 \mu \mathrm{g}$ of biotin twice daily for eight weeks. Assignment to biotin supplement or placebo was randomized and not stratified. The biotin supplement and the placebo (as dextrose) were given in identical coded bottles. Participants were instructed to take one capsule in the morning and one capsule in the evening daily for eight weeks and continue to take any other prescribed medications. All participants and researchers were blinded to the treatment until all data were collected. Compliance was assessed by examination of study bottles returned to the study coordinators at the end of participation in the study. Any participant who did not consume all biotin supplements or all placebos was deemed noncompliant $(n=1)$, and those data were excluded from the analysis.

\section{Statistical analysis}

Significance was chosen as $P$ value $\leq 0.05$. Testing for significance in the difference in mean values of biotin indicators for participants in Study 1 with and without RLS (Table 1) was conducted using MannWhitney $\mathrm{U}$ test, a non-parametric test for two independent samples. In Study 2, for all categorical variables (Table 2), Fisher's exact test was used to determine the significance of the differences in the proportions for the biotin and placebo arms. In Study 2, the significance of the differences of group mean PCC and AC of PCC before and after treatment with biotin or placebo was tested using the one-tailed, paired $t$ test. For all analyses, statistical testing was conducted using Kaleidagraph (Synergy Software, Reading, PA).

\section{Results}

\section{Study 1}

A total of 59 participants receiving dialysis (49 hemodialysis, 10 
peritoneal dialysis) were enrolled and provided information about the diagnosis of RLS and were evaluated by study personnel for an IRLS score (Figure 1). Demographic characteristics for the subgroups with and without RLS are provided in table 1 . The RLS group trended to more women and to older individuals. The duration of dialysis and the prevalence of diabetes were similar between the two groups.

Technically adequate samples for quantitation of lymphocyte AC of PCC were available in 49 of the 60 participants. Of those 49 , 30 participants had a diagnosis of RLS; mean \pm 1 SD of IRLS score $=18 \pm 7$. Each of the eighteen who were not diagnosed with RLS had IRLS score $=0$. Thus, the incidence of RLS in this population of ESRD patients is similar to what has been reported previously [1,2]. In Study 1 , patients with $(n=30)$ were more likely than those without $(n=19)$ Restless Legs Syndrome to be biotin deficient as judged by increased activation coefficient of propionyl-CoA carboxylase; mean ( $\pm 1 \mathrm{SD})$ was $1.17 \pm 0.65$ vs. $0.87 \pm 0.39$, respectively $(P=0.007)$. Mean AC of PCC was significantly greater in the RLS subgroup (Table 1 ). In the RLS subgroup, eight of 30 (26\%) participants had activation coefficients of greater than two standard deviations above the mean of the normal population compared to only three of $19(16 \%)$ in the subgroup without RLS symptoms $(P=0.79)$. Although this is not different, these observations suggest that the RLS subgroup could possibly contain a subpopulation of individuals with importantly impaired intracellular biotin status.

\begin{tabular}{|l|c|c|c|}
\hline & RLS & No RLS & $P$ value \\
\hline Female & $14 / 30$ & $5 / 19$ & 0.41 \\
\hline Age (years, mean \pm SD) & $64.0 \pm 13.1$ & $56.2 \pm 12.4$ & 0.87 \\
\hline Prevalence of Diabetes & $15 / 30$ & $7 / 19$ & 0.61 \\
\hline Peritoneal Dialysis & $5 / 6$ & $1 / 6$ & 0.24 \\
\hline $\begin{array}{l}\text { Duration of Dialysis (years, } \\
\text { mean } \pm \text { SD) }\end{array}$ & $3.1 \pm 2.3$ & $2.4 \pm 1.3$ & 0.18 \\
\hline $\begin{array}{l}\text { AC of PCC } \\
\text { (mean } \pm \text { SD) }\end{array}$ & $\begin{array}{c}1.17 \pm 0.65 \\
\mathrm{n}=30\end{array}$ & $\begin{array}{c}0.87 \pm 0.39 \\
\mathrm{n}=19\end{array}$ & 0.007 \\
\hline
\end{tabular}

Table 1: Demographic Characteristics and Activation Coefficient (AC) for Propionyl-CoA Carboxylase (PCC) for Participants in Study 1 with and Without Restless Legs Syndrome (RLS).

\begin{tabular}{|l|c|c|c|}
\hline & Biotin group & Placebo group & P value \\
\hline Female & $5 / 16$ & $5 / 15$ & 1.0 \\
All Participants & $3 / 8$ & $3 / 7$ & 0.7 \\
\hline Technical Adequate AC of PCC & $60 \pm 12$ & $63 \pm 10$ & 0.6 \\
\hline $\begin{array}{l}\text { Age (years, mean } \pm \text { SD) } \\
\text { All Participants }\end{array}$ & $64 \pm 9$ & $64 \pm 7$ & 0.9 \\
\hline Technical Adequate AC of PCC & $5 / 16$ & $9 / 15$ & 0.4 \\
\hline $\begin{array}{l}\text { Prevalence of Diabetes } \\
\text { All Participants }\end{array}$ & $5 / 7$ & $4 / 7$ & 0.7 \\
\hline Technical Adequate AC of PCC & $3 / 16$ & $1 / 15$ & 0.7 \\
\hline Type of Dialysis (peritoneal/total) All & $2 / 2$ & $2 / 5$ & 0.58 \\
\hline Participants & & & \\
\hline Technical Adequate AC of PCC & $2.8 \pm 2.7$ & $3.2 \pm 2.4$ & 0.6 \\
\hline $\begin{array}{l}\text { Duration of Dialysis } \\
\text { (years, mean } \pm \text { SD) } \\
\text { All Participants } \\
\text { Technical Adequate AC of PCC }\end{array}$ & $3.1 \pm 2.6$ & $4.6 \pm 2.1$ & 0.3 \\
\hline $\begin{array}{l}\text { Died (between enrollment and } \\
\text { completion) }\end{array}$ & $2 / 21$ & $1 / 16$ & 1.0 \\
\hline \multicolumn{1}{|l|}{ Table 2: Demographics and Comorbidity Prevalence for Study 2 Groups. }
\end{tabular}

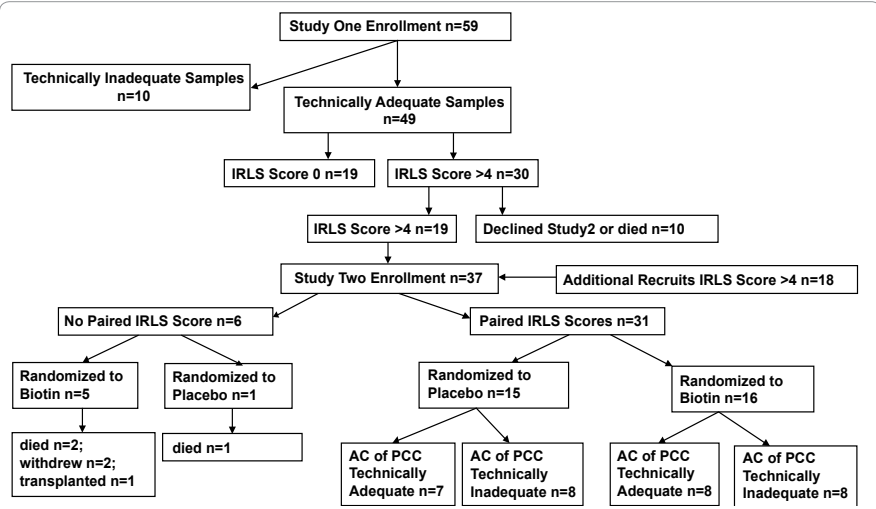

Figure 1: Flow diagram for enrollment and retention of participants in Study 1 and Study 2.

\section{Study 2}

Randomized, Double-Blind, Placebo-Controlled Trial of Biotin Supplementation in RLS and ESRD Ninteen participants in Study 1 with RLS agreed to participate in Study 2. An additional 18 ESRD patients with RLS also agreed to participate in Study 2. Of these 37, only 31 completed the two sets of questionnaires required to calculate the paired IRLS scores (before and after treatment with biotin or placebo). The six lost to follow up for the following reasons: 2 withdrew, 3 died, and 1 received a kidney transplant (Figure 1). To assess for bias introduced by randomization to biotin or placebo, we compared the incidence of RLS and the incidence of biotin deficiency prior to supplementation in several demographic subgroups: gender, age, diagnosis of diabetes, and duration of dialysis. We found no significant differences between the treatment group and placebo group for these demographics (Table 3).

Mean IRLS score decreased with biotin supplementation (Figure 2A). However, mean IRLS score also decreased with placebo. The net change in IRLS score was not different between biotin and placebo.

Of the 31 , only 15 participants had technically adequate samples for AC of PCC from both before and after treatment with biotin or placebo: eight of the 16 were treated with biotin and seven of the 15 were treated with placebo. Mean AC of PCC decreased with biotin supplementation but not with placebo (Figure 2B).

We were concerned that some of the differences observed in Study 2 might have resulted from differences in severity of illness, comorbidity prevalence, or type of dialysis within the two treatment arms or within the subgroups of each arm that had technically adequate AC of PCC samples. However, examination revealed that the treatment groups were not different with respect to gender, age, prevalence of diabetes, type of dialysis, or duration of dialysis. There were no differences in the number of the participants that did not complete the study due to death (Table 2). Similarly, the subgroups for which technically adequate samples were available for determination of AC of PCC were not different with respect to gender, age, prevalence of diabetes, or duration of dialysis (Table 3).

Other than the reported difference in AC of PCC, no clinical or laboratory findings attributable to biotin deficiency or to biotin toxicity were observed in either the biotin group or the placebo group.

\section{Discussion}

This study provides evidence that biotin status as assessed by the Activation Coefficient (AC) of the biotin-dependent enzyme PCC is 

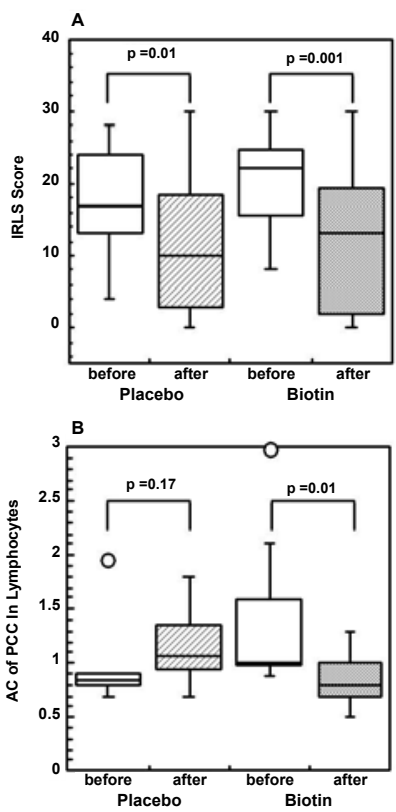

Figure 2: Effect of Biotin Supplementation on Restless Legs Syndrome Score (A) and Biotin Status (B). International Restless Legs Study Group [16] scores are depicted as box-whisker plots; the upper and lower limits of the box depict the 75th and 25th quartiles, the midline depicts the medians, and the whiskers depict the ranges. IRLS scores decreased with biotin supplementation $(P=0.001, \mathrm{n}=16)$ but also decreased with placebo $(P=$ $0.01, n=15)$; the decrease was not significantly different between biotin and placebo. Lymphocytes (Figure 2B) were isolated from Study 2 participants before and after supplementation with biotin or placebo for 8 weeks. Isolated lymphocytes were incubated with and without $10 \mathrm{nmol} / \mathrm{L}$ of biotin before the activity of propionyl-CoA carboxylase (PCC) was determined. The boxwhisker plots (as described in $2 \mathrm{~A}$ ) depict the change in PCC activity after biotin incubation; in addition, outlier values greater than the upper quartile + $1.5^{\star} \mathrm{IQD}$ (inter-quartile distance) or less than the lower quartile $-1.5^{*} \mathrm{IQD}$ are depicted as individual points. The activation coefficient decreased $(P=0.01)$ with biotin supplementation $(n=8)$ but not with placebo $(n=7)$.

\begin{tabular}{|l|c|c|c|}
\hline & Biotin group & Placebo group & P value \\
\hline $\begin{array}{l}\text { Female } \\
\text { RLS }\end{array}$ & $5 / 9(55 \%)$ & $5 / 12(45 \%)$ & 0.55 \\
AC of PCC & $3 / 7$ & $3 / 7$ & 0.71 \\
\hline Age & & & \\
(years, mean \pm SD) & $64 \pm 8$ & $64 \pm 9$ & 0.94 \\
RLS & $64 \pm 9$ & $64 \pm 7$ & 0.94 \\
AC of PCC & $5 / 9(55 \%)$ & $9 / 12(75 \%)$ & 0.38 \\
\hline Diabetes & $5 / 7$ & $4 / 7$ & 0.66 \\
\hline RLS & & & \\
AC of PCC & $3.5 \pm 2.6$ & $3.2 \pm 2.4$ & 0.999 \\
\hline Duration of Dialysis & & $4.6 \pm 2.1$ & 0.28 \\
\hline (years, mean \pm SD) & $3.1 \pm 2.6$ & & \\
\hline RLS & & & \\
\hline
\end{tabular}

Table 3: Demographic Characteristics for Study 2 Subgroups.

significantly impaired in individuals with ESRD who have RLS compared with individuals with ESRD who do not have RLS. This difference was observed despite daily, standard-of-care, oral supplementation with biotin at five to ten times the Adequate Intake (AI). Based solely on this observed association, inferring a causal relationship between biotin deficiency and RLS in ERSD would be problematic, particularly in view of the small number of Study 1 participants and in view of the subjective nature of RLS symptoms. Accordingly, we conducted a randomized-assignment, double-blinded, placebo-controlled study of the effect of high dose biotin supplementation on IRLS score and on the AC of PCC. The mean activation coefficient of PCC in the biotintreatment group improved significantly and did not change in the placebo group. Moreover, the IRLS score improved substantially in the group receiving $10,000 \mu \mathrm{g}$ of biotin daily for eight weeks. However, a smaller but still highly significant improvement in IRLS score was also observed in the placebo group; the decrease was not significantly different between biotin and placebo. On balance, these data are only moderately supportive of the inference that functional biotin status is suboptimal in ESRD patients receiving dialysis and that there is a therapeutic role for supplementation with high doses of biotin.

Additional important limitations to confidence in our inferences are overall small sample size and the relatively low rate at which individuals completed Study 2. Due to death (8\%), withdrawal from the study (5\%), and technically inadequate blood samples for initial determination of AC of PCC (52\%), only 15 of 37 (41\%) participants who were initially recruited for the study completed all of Study 2, complying with the biotin or placebo treatment and providing initial and final IRLS score assessment. This low rate of study completion allows for the possibility that sampling bias may have inadvertently occurred. However, examination of the demographic and comorbidity prevalence data in the subgroups (Table 2) yielded no evidence of that bias.

This study also did not address either the mechanism leading to impaired biotin status or to biotin-responsive RLS symptoms. Several pathogenic mechanisms appear plausible. Although no quantitation of the rate of removal of biotin during dialysis has been published, we speculate that biotin is likely to be removed by hemodialysis and peritoneal dialysis on the basis of molecular weight (244 Daltons) and minimal binding to plasma macromolecules (presumably proteins) [20]. Intestinal uptake of biotin might also be impaired in ESRD. In a rat model of ESRD achieved by a complete nephrectomy of one kidney and two-thirds segmental infarction of the remaining kidney (5/6 nephrectomy), Said and coworkers reported that ESRD suppresses biotin transport in the small intestine [21]. The $\mathrm{V}_{\max }$ for biotin transport in inverted jejunal sacs was reduced by about twothirds, without a significant change in $\mathrm{K}_{\mathrm{M}}$. Moreover, in brush border membrane vesicles made from the jejunum, $\mathrm{V}_{\max }$ of biotin transport was reduced by about one third in this ESRD model. Pathogenesis likely involved alterations in gene expression or transporter stability for the sodium dependant multi-vitamin transporter, SMVT [21]. If a similar pathogenic process occurs in patients with ESRD despite dialysis, net intestinal uptake of biotin would likely be reduced. This impaired intestinal biotin uptake appears to be analogous to the marked downregulation in the expression of folate and thiamin transporters in the intestine of the 5/6 nephectomized rat [22]. Of note, transport of folate and thiamin were also down regulated in somatic tissue such as heart, liver, and brain, potentially leading to impaired cellular homeostasis of these essential micronutrients despite normal or near normal plasma levels. Theoretically, a similar down regulation of biotin transport into somatic tissues might occur in ESRD.

In addition to these pathogenic processes arising directly from ESRD and dialysis, restriction to lower protein diets might restrict biotin intake [23]. More frequent use of antibiotics could suppress the intestinal biome and hence the production and release of biotin by intestinal microbes; the contribution of gut bacteria to net absorbed biotin remains unknown. A higher incident of therapy with medications that accelerate biotin biotransformation to inactive metabolites (e.g., 
Citation: Moretti H, Matthews N, Anthony Lakatua JD, Keeling-Hathaway T, Mock DM (2012) Biotin Deficiency as a Target for Treating Restless Legs Syndrome in Chronic Dialysis Patients. J Nephrol Therapeut 2: 128. doi:10.4172/2161-0959.1000128

Page 5 of 5

antiepileptics) might also contribute to reducing biotin status in ESRD [24-27].

We offer no mechanisms for 1) the RLS symptoms reported by these individuals with ESRD, 2) the biotin responsiveness of their RLS, or 3 ) the striking placebo effect on RLS symptoms. The contribution of uremic peripheral neuropathy to RLS in ESRD and during dialysis remains unclear.

We saw no clinical findings attributable to biotin toxicity. The National Academy of Sciences has not yet established a tolerable upper limit for biotin [15]. However, no toxicity has been reported even with doses of biotin up to $200,000 \mu \mathrm{g}$, a dose that has been given orally for treatment of inborn errors of metabolism. Supplements of 10,000 $\mu \mathrm{g}$ of biotin per day have been given intramuscularly for a year with no reported side effects and have been reported to improve symptoms of peripheral neuropathy in ESRD $[7,8]$. Further, the catabolites of biotin are not active and apparently do not interfere with the attachment of biotin to apocarboxylases [27]. Thus, excess biotin is not likely to interfere with normal biotin function.

\section{Practical Application}

RLS treatment options are limited in dialysis patients and often have inadequate response. Our findings of an apparent association between biotin status and RLS and of a modest therapeutic effect of biotin deserve further study. In that context, biotin in high doses was found to be safe, beneficial, and well tolerated.

\section{Acknowledgement}

This work was supported by National Institutes of Health, Institute of Diabetes Digestive, and Kidney Diseases R37 DK036823-026, and R37 DK036823-26S1 (DMM) and Saint Patrick Hospital Foundation, and Fraternal order of Eagles grant (HM)

The authors have no conflict of interest or any relevant financial interest associated with this study.

HM acknowledges the gift of biotin provided by Hillestad Pharmaceuticals.

The authors were exclusively responsible for study design, sample collection, analysis and interpretation of the data, and preparation of this manuscript.The authors appreciate the technical expertise of Shan Zhu, MD, for the measurement of propionyl-CoA carboxylase activities.

\section{References}

1. Siddiqui S, Kavanagh D, Traynor J, Mak M, Deighan C, et al. (2005) Risk factors for restless legs syndrome in dialysis patients. Nephron Clin Pract 101: c155-160.

2. Winkelmann J, Polo O, Provini F, Nevsimalova S, Kemlink D, et al. (2007) Genetics of restless legs syndrome (RLS): State-of-the-art and future directions. Mov Disord 22: S449-S458.

3. Ram S, Seirawan H, Kumar SK, Clark GT (2010) Prevalence and impact of sleep disorders and sleep habits in the United States. Sleep Breath 14: 63-70.

4. Allen RP, Picchietti D, Hening WA, Trenkwalder C, Walters AS, et al. (2003) Restless legs syndrome: diagnostic criteria, special considerations, and epidemiology. A report from the restless legs syndrome diagnosis and epidemiology workshop at the National Institutes of Health. Sleep Med 4: 101 119.

5. Sloand JA, Shelly MA, Feigin A, Bernstein P, Monk RD (2004) A double-blind placebo-controlled trial of intravenous iron dextran therapy in patients with ESRD and restless legs syndrome. Am J Kidney Dis 43: 663-670.

6. Makoff R, Gonick H (1999) Renal failure and the concomitant derangement of micronutrient metabolism. Nutr Clin Pract 14: 238-246.

7. Yatzidis H, Koutsicos D, Agroyannis B, Papastephanidis C, Francos-Plemenos $M$, et al. (1984) Biotin in the management of uremic neurologic disorders. Nephron 36: 183-186.

8. Yatzidis H, Koutsicos D, Alaveras AG, Papastephanidis C, Frangos-Plemenos
M (1981) Biotin for neurologic disorders of uremia. N Engl J Med 305: 764

9. Lutz-Mizar J (2004) Biotin in the management of uremic neurologic disorders Diagnostic Nutrition Network 13: 1-5.

10. Pacheco-Alvarez D, Solórzano-Vargas RS, Del Río AL (2002) Biotin in metabolism and its relationship to human disease. Arch Med Res 33: 439-447.

11. Mock D, Henrich C, Carnell N, Mock N, Swift L (2002) Lymphocyte propionylCoA carboxylase and accumulation of odd-chain fatty acid in plasma and erythrocytes are useful indicators of marginal biotin deficiency small star, filled. J Nutr Biochem 13: 462

12. Stratton SL, Bogusiewicz A, Mock MM, Mock NI, Wells AM, et al. (2006) Lymphocyte propionyl-CoA carboxylase and its activation by biotin are sensitive indicators of marginal biotin deficiency in humans. Am J Clin Nutr 84: 384-388.

13. Ouseph R, Hutchison CA, Ward RA (2008) Differences in solute removal by two high-flux membranes of nominally similar synthetic polymers. Nephrol Dial Transplant 23: 1704-1712.

14. Gondouin B, Hutchison CA (2011) High cut-off dialysis membranes: current uses and future potential. Adv Chronic Kidney Dis 18: 180-187.

15. Research Council (1998) Dietary reference intakes for thiamin, riboflavin, niacin, vitamin B6, folate, vitamin B12, pantothenic acid, biotin, and choline. National Academy Press, Washington DC.

16. Walters AS, LeBrocq C, Dhar A, Hening W, Rosen R, et al. (2003) Validation of the International Restless Legs Syndrome Study Group rating scale for restless legs syndrome. Sleep Med 4: 121-132.

17. Zempleni J, Mock DM (2000) Utilization of biotin in proliferating human lymphocytes. J Nutr 130: 335S-337S

18. Mock DM (2009) Marginal biotin deficiency is common in normal human pregnancy and is highly teratogenic in mice. J Nutr 139: 154-157.

19. Stratton SL, Henrich CL, Matthews NI, Bogusiewicz A, Dawson AM, et al (2012) Marginal biotin deficiency can be induced experimentally in humans using a cost-effective outpatient design. J Nutr 142: 22-26.

20. Mock DM, Malik MI (1992) Distribution of biotin in human plasma: most of the biotin is not bound to protein. Am J Clin Nutr 56: 427-432.

21. Said HM, Vaziri ND, Oveisi F, Hussienzadha S (1992) Effect of chronic rena failure on intestinal transport of biotin in the rat. J Lab Clin Med 120: 471-475.

22. Bukhari FJ, Moradi H, Gollapudi P, Ju Kim H, Vaziri ND, et al. (2011) Effect of chronic kidney disease on the expression of thiamin and folic acid transporters. Nephrol Dial Transplant 26: 2137-2144.

23. Velázquez A, Martín-del-Campo C, Báez A, Zamudio S, Quiterio M, et al. (1989) Biotin deficiency in protein-energy malnutrition. Eur J Clin Nutr 43: 169-173.

24. Krause KH, Berlit $\mathrm{P}$, Bonjour JP (1982) Impaired biotin status in anticonvulsant therapy. Ann Neurol 12: 485-486.

25. Said HM, Redha R, Nylander W (1989) Biotin transport in the human intestine: inhibition by anticonvulsant drugs. Am J Clin Nutr 49: 127-131.

26. Mock DM, Dyken ME (1997) Biotin catabolism is accelerated in adults receiving long-term therapy with anticonvulsants. Neurology 49: 1444-1447.

27. Mock DM, Matthews NI (2011) Biotin and pantothenic acid: Biochemical, Physiological and Molecular Aspects of Human Nutrition. Elsevier. 